\title{
Assessment of General MRI, Morphological and Signal Characteristics of Benign and Malignant Ovarian Tumors
}

\author{
Naima Mannan ${ }^{1 *}$, Mukesh Mittal $^{2}$ \\ ${ }^{1 *}$ Professor, ${ }^{2}$ Assistant Professor, \\ Department of Radiodiagnosis, SMS Medical College, Jaipur, Rajasthan, INDIA.
}

\begin{abstract}
Introduction: Characterization of an ovarian mass is of the utmost importance in the preoperative evaluation of an ovarian neoplasm. In view of this, present study was carried to evaluate the diagnostic accuracy of diffusion weighted MRI in differentiating pathologically confirmed benign and malignant ovarian masses.

Material and Methods: This hospital based observational descriptive study comprised of 50 patients diagnosed with ovarian masses clinically or on ultrasonography were evaluated with pelvic MRI. Statistical Analysis was done using IBM SPSS Statistics 21. Surgical pathological findings were used as the reference standard for assessment of ovarian tumors. Non-parametric MRI variables were analyzed using Chi square test or Fischer's exact test, whichever was applicable. $P$ value of less than 0.05 was considered for statistical significance.

Results: There was no difference in maximal diameter between benign $(82.5 \pm 41.23 \mathrm{~mm})$ and malignant $(96.3 \pm 28.17)$ adnexal masses. Low signal intensity of solid component on T2W and DW Images was more frequent in benign masses than in malignant masses $(p$ value $=0.002$ and 0.001 respectively).
\end{abstract}

\section{INTRODUCTION}

Ovarian masses are a common finding in clinical practice and may be diagnosed incidentally or in symptomatic patients. ${ }^{1}$ Although they represent similar clinical and radiologic features, however, predominant or specific imaging features may be present in some types of ovarian tumors. Characterization of an ovarian mass is of the very important in the preoperative evaluation of an ovarian neoplasm $^{2}$ and represents a diagnostic challenge in the preoperative setting in order to plan adequate therapeutic procedures and may influence patient's management, ${ }^{1}$ as well as enables the surgeon to anticipate carcinoma of the ovary before the operation so that adequate procedures can be planned. ${ }^{2}$

Magnetic resonance (MR) imaging may provide useful information for the characterization of ovarian masses as non-neoplastic or neoplastic, and, in the latter case, as benign or malignant. MR imaging can distinguish several types of tissue and fluid from their signal intensity patterns. The signal intensities of a tumour depend upon the presence, type and extent of solid tumour and cystic components of the mass. Tumours with predominantly smooth muscle or fibrotic component, such as fibroma, fibrothecoma, cystadenofibroma, Brenner tumour and leiomyoma, have low to intermediate signal on T2-weighted images. Predominantly or uniformly low signal intensity within a lesion is therefore a feature of benign tumours. ${ }^{3}$
Conclusion: The most significant criteria for predicting malignancy were intermediate $\mathrm{T} 2$ and high DW signal (PLR = 4.33) followed by papillary projections (PLR $=3.89$ ). Our study demonstrated that diffusion-weighted MR imaging (DWI) combined with classical T2-weighted imaging is an accurate tool to assess the nature of complex adnexal masses depicted by ultrasonography (US).

Keywords: Magnetic resonance (MR) imaging; Ovarian masses.

\section{*Correspondence to:}

Dr. Naima Mannan, Professor, Department of Radiodiagnosis, SMS Medical College, Jaipur, Rajasthan, INDIA.

Article History:
Received: 21-04-2016, Revised: 29-04-2016, Accepted: 13-05-2016
\begin{tabular}{|l|c|}
\hline \multicolumn{2}{|c|}{ Access this article online } \\
\hline Website: & Quick Response code \\
www.ijmrp.com & \\
\hline DOl: & \\
10.21276/jmrp.2016.2.3.035 & \\
\hline
\end{tabular}

In view of this, present study was carried to evaluate the diagnostic accuracy of diffusion weighted MRI in differentiating pathologically confirmed benign and malignant ovarian masses.

\section{MATERIAL AND METHODS}

This hospital based observational descriptive study was conducted in Department of Radio-diagnosis and Modern imaging of SMS Medical College, Jaipur, Rajasthan, supported by departments of Obstetrics and Gynecology and Surgery. The main source of data for this study was patients from the Department of Obstetrics and Gynecology and Department of Surgery. A total of 50 patients diagnosed with ovarian masses on Ultrasound or on clinical examination were evaluated with conventional MRI, diffusion-weighted MRI and contrast enhanced MRI. Surgical/Histopathological results served as the gold standard. In case of bilateral ovarian masses with same histopathological diagnosis, most complex mass was used for analysis.

Inclusion criterion

- Patients referred from Surgery \& Surgical Oncology and Obstetrics and Gynecology, suspected to have ovarian masses willing to undergo Contrast Enhanced and Diffusion Weighted MRI.

- Patients who gave informed written consent. 


\section{Exclusion criterion}

- Patients suspected of adnexal masses in which the ultrasongraphy was normal and any other pathology causing symptoms was found

- Non availability of the histopathological examination report.

- Patients with complex or indeterminate adnexal masses that exhibit a high T1 signal on conventional MR sequences (endometrioma or dermoid cyst)

- Patient with MR incompatible devices or implants.

- Patients with Claustrophobia.

- Uncooperative or unstable patients.

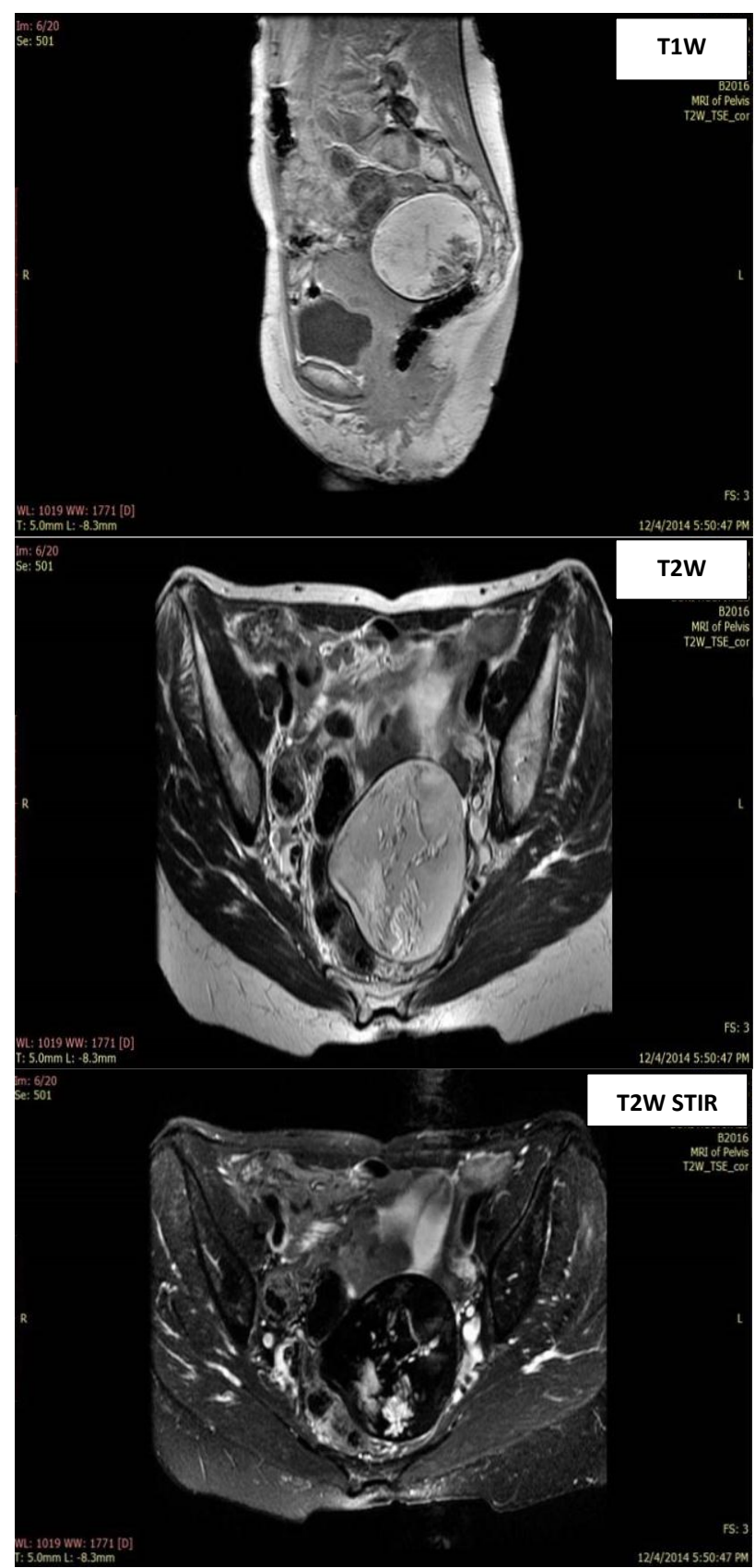

Fig 1: This case illustrates a large ovarian mass that was hyperintense on $\mathrm{T} 1$ and $\mathrm{T} 2$ with suppression of signal on $\mathrm{T} 2 \mathrm{~W}$ STIR. Typical MRI features of a large

\section{MRI protocol}

All patients suspected of adnexal mass clinically or on ultrasonography were evaluated with pelvic MRI. All subjects underwent MRI with a 3T MR unit (Philips Ingenia). The imaging protocol involved axial non-contrast T1-weighted (TR/TE, 400-
600/10-14 ms), axial T2-weighted (TR/TE, 4,000-6,000/100-120 $\mathrm{ms}$ ) imaging using the following parameters: slice thickness, $4-5$ $\mathrm{mm}$; gap, 0-1 mm; field of view (FOV), 32 to $42 \mathrm{~cm}$; matrix, $256 \times$ 256.

Sagittal T2-weighted (TR/TE, 3,000-6,000/100-110 ms) fast spin echo imaging with chemical shift-selective fat saturation pulse were also performed, as well as post-contrast enhanced axial and sagittal T1-weighted imaging using parameters as described above.

Diffusion weighted MRI were acquired in the axial plane prior to administration of contrast medium using a single-shot echoplanar imaging sequence (TR/TE effective range, 8,000-10,000/70-100; slice thickness/ intersection gap, $5 / 1.5 \mathrm{~mm}$; FOV, 32 to $42 \mathrm{~cm}$; matrix, $128 \times 128$; excitation). A b-value of 0 and of $1,000 \mathrm{~s} / \mathrm{mm}^{2}$ were applied in three orthogonal $(Z, Y$, and $X)$ directions. Conventional MRI and DWI imaging data then were analyzed.

\section{Data calculation and MR Image analysis}

MR Images were evaluated and morphological and diffusion characteristics recorded as per the proforma. The solid and cystic component were identified according to a previously established classification by Timmerman et al $^{4}$ that classified papillary projections, thick septa $>3 \mathrm{~mm}$ and any enhancing solid portion together as solid component.

Signal intensity of solid components was evaluated on T2weighted MR images relative to outer myometrium , and classified as "low" when lower in signal, and "intermediate" when the signal was equal to or greater than the outer myometrium. The presence of ascites or peritoneal implants associated with the tumor was recorded.

Statistical Analysis was done using IBM SPSS Statistics 21. Surgical pathological findings were used as the reference standard for assessment of ovarian tumors. Non-parametric MRI variables were analyzed using Chi square test or Fischer's exact test, whichever was applicable. $P$ value of less than 0.05 was considered for statistical significance.

Table 1: Distribution of Patients In Relation To Age

\begin{tabular}{lcc}
\hline Age & Total & Percentage \\
\hline$<35$ & 8 & 16 \\
$35-50$ & 16 & 32 \\
$>50$ & 26 & 52 \\
Grand Total & 50 & 100 \\
\hline
\end{tabular}

\section{RESULTS}

Distribution of patients in relation to age is given in table 1 . There was no difference in maximal diameter between benign $(82.5 \pm 41.23 \mathrm{~mm})$ and malignant $(96.3 \pm 28.17)$ adnexal masses. Nine masses were purely cystic and all these cystic masses were benign. Most of predominantly cystic masses were malignant $(p=0.01)$. Papillary projections or solid portions were less common in benign than malignant masses ( $p$ value 0.012 and 0.002 respectively). Solid portions were found in only $42 \%$ of benign masses as compared to $89 \%$ in malignant masses. Ascites and peritoneal implants were less frequently associated with benign masses $(p=0.017)$. Ascites was found in 11 patients. None of the benign masses was associated with peritoneal implants (Table 2 and 3). 
Table 2: General MRI Characteristics of Benign and Malignant Ovarian Tumors

\begin{tabular}{lccc}
\hline MRI Features & Benign $(\mathbf{n}=\mathbf{3 3})$ & Malignant $(\mathbf{n}=17)$ & P Value \\
\hline Max Diameter (mean, range) & $82.5 \mathrm{~mm} \pm 41.23$ & $96.3 \mathrm{~mm} \pm 28.17$ & 0.76 \\
Multilocularity & $13 / 33(40 \%)$ & $6 / 17(36 \%)$ & 0.83 \\
Loculi with different signal intensity & $5 / 33(15 \%)$ & $4 / 17(23 \%)$ & 0.37 \\
Ascites or Peritoneal Implants & $11 / 33(30 \%)$ & $12 / 17(71 \%)$ & 0.017 \\
\hline
\end{tabular}

Table 3: Morphological Characteristics of Solid Component* on MRI (Septa/Papillary Projection/Solid Portion)

\begin{tabular}{lccc}
\hline MRI Features & Benign (n=33) & Malignant $(\mathbf{n}=\mathbf{1 7})$ & P Value $^{\text {** }}$ \\
\hline Solid and Cystic Components & & & \\
Predominantly cystic & $18 / 33(54 \%)$ & $14 / 17(83 \%)$ & 0.01 \\
Purely Cystic & $9 / 33(27 \%)$ & $0 / 17(0 \%)$ & $<0.001$ \\
Thickened septa & $6 / 33(18 \%)$ & $5 / 17(30 \%)$ & 0.475 \\
Papillary Projections & $4 / 33(12 \%)$ & $8 / 17(47 \%)$ & 0.012 \\
Solid Portion & $14 / 33(42 \%)$ & $15 / 17(89 \%)$ & 0.002 \\
\hline
\end{tabular}

${ }^{*}$ Total No of Benign Lesions with solid component $=21$

*Total No of Malignant Lesions with solid component $=17$

** $P$ value from Fischer's exact test

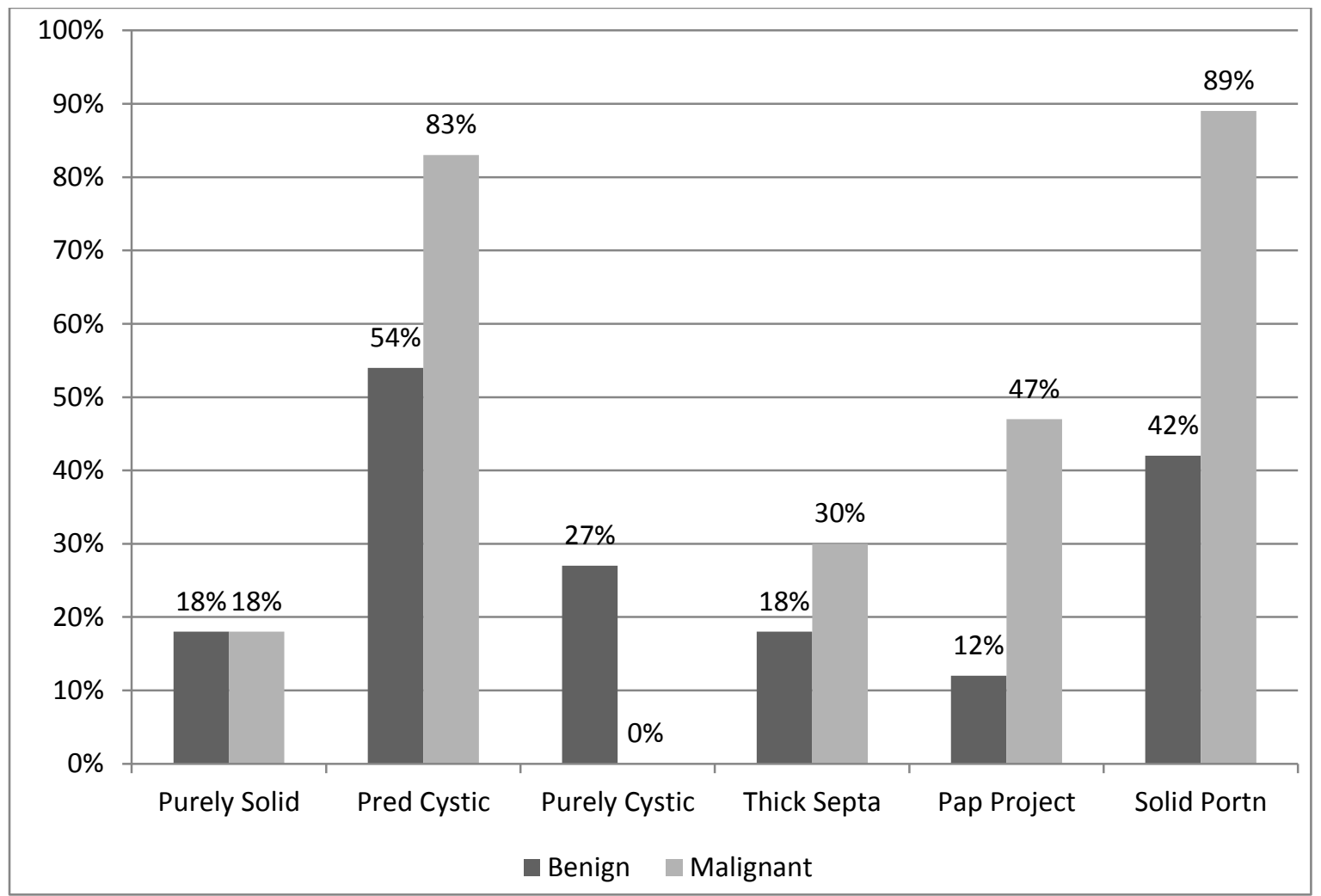

Graph 1: Morphological Characteristics of Solid Component* on MRI (Septa/Papillary Projection/Solid Portion)

Table 4: Signal Characteristics of Solid Component * (Septa/Papillary Projection/Solid Portion)

\begin{tabular}{lccc}
\hline Signal Intensity & Benign ( $\mathbf{n}=21)$ & Malignant $(\mathbf{n}=17)$ & $\mathbf{P}^{\text {value }} \mathbf{e}^{* *}$ \\
\hline Low T2 signal & $13 / 21(61 \%)$ & $2 / 17(12 \%)$ & 0.002 \\
Intermediate T2 signal & $8 / 21(38 \%)$ & $15 / 17(88 \%)$ & 0.002 \\
Low DW signal & $15 / 21(72 \%)$ & $3 / 17(18 \%)$ & 0.001 \\
High DW signal & $6 / 21(29 \%)$ & $14 / 17(82 \%)$ & 0.001 \\
Low T2 + Low DW signal & $11 / 21(52 \%)$ & $2 / 17(12 \%)$ & 0.015 \\
Intermediate T2+ High DW & $4 / 21(19 \%)$ & $14 / 17(82 \%)$ & $<0.001$ \\
\hline
\end{tabular}

${ }^{*}$ Total No of Benign Lesions with solid component $=21$

${ }^{*}$ Total No of Malignant Lesions with solid component $=17$

${ }^{* *} P$ value from Fischer's exact test 


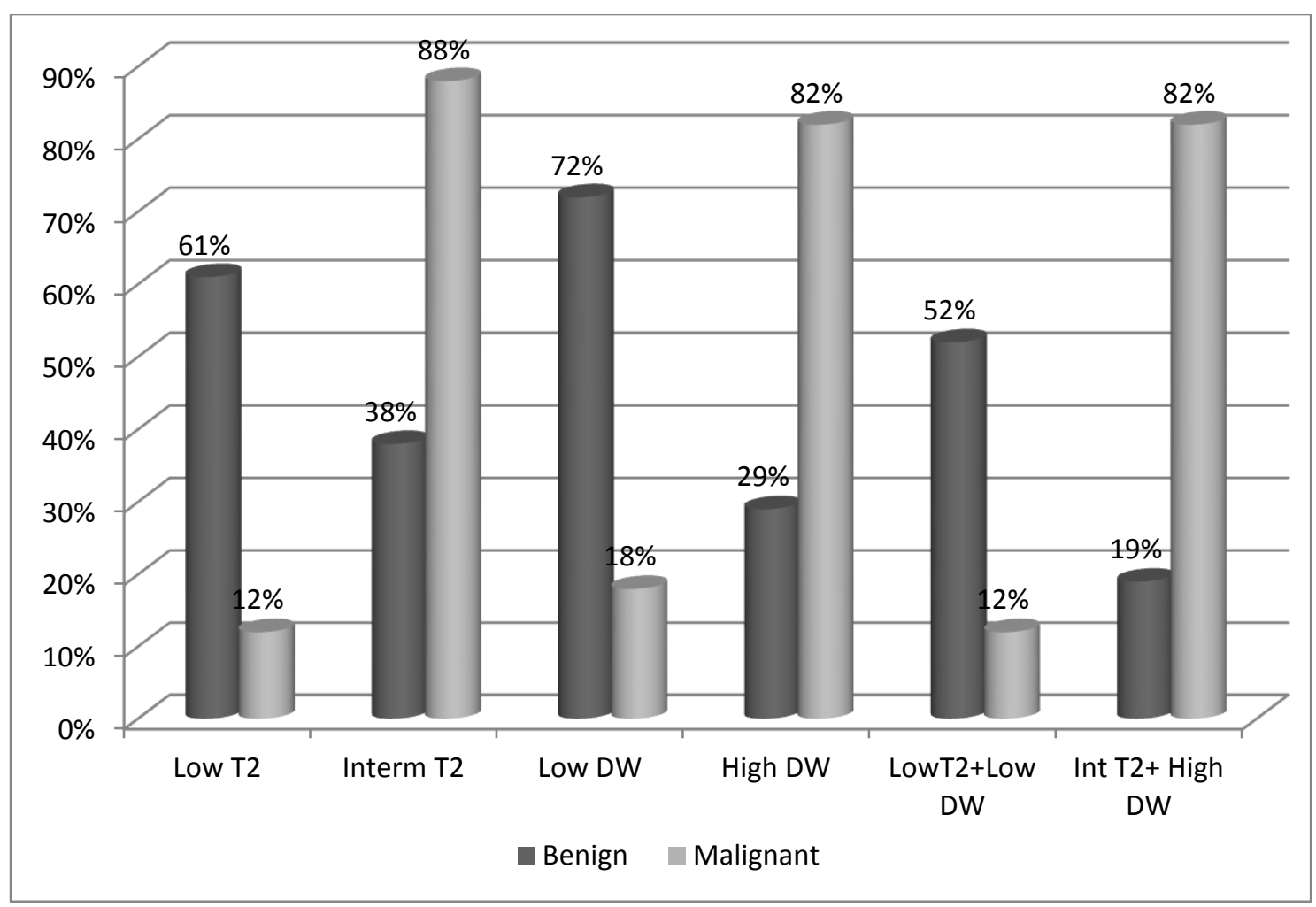

Graph 2: Signal Characteristics of Solid Component (Septa/Papillary Projection/Solid Portion)

Low signal intensity of solid component on T2W and DW Images was more frequent in benign masses than in malignant masses ( $p$ value $=0.002$ and 0.001 respectively). Among the 21 benign lesions with solid component two ovarian fibromas, two mucinous cystadenoma and two serous cystadenoma showed high DW signal. Among the malignant tumors only three tumors, one granulosa-sertoli cell tumor, one metastasis and one serous cystadenocarcinoma showed low DW signal. Intermediate T2 and high DW signal was more common in malignant masses ( $p$ value $=0.002$ and 0.001 respectively). Combination of intermediate $\mathrm{T} 2$ and High DW signal had most significant association with malignant masses ( $p$ value $<0.001$ ) (table 4 ).

The most significant criteria for predicting malignancy were intermediate $\mathrm{T} 2$ and high DW signal (PLR $=4.33$ ) followed by papillary projections $(P L R=3.89)$. The most significant criteria for excluding malignancy were low DW signal (NLR $=2.88)$ followed by combination of low T2 and low DW signal (NLR $=1.85$ ).

\section{DISCUSSION}

Our study suggests that MRI with DWI may have a role in the preoperative evaluation of these women, to determine therapeutic strategy, including the possibility of expectant management, the feasibility of laparoscopy and conservative surgery. Our study confirms that low T2 signal intensity within a solid component is a good criterion for predicting benignity of ultrasonographically indeterminate or complex masses. ${ }^{4-6}$ In addition, our study suggests that DW signal intensity within the solid component should help radiologists characterize these masses, sometimes misdiagnosed with conventional imaging. ${ }^{4}$ Moreover, our study demonstrates the gain of combining the conventional T2-weighted sequences with diffusion-weighted sequence, since a tumor exhibiting both low T2 and low DW signal intensity within the solid component was almost always benign. Thus, our results support the use of imaging follow-up for patients with low T2 and DW signal intensity within the solid component of a suspicious adnexal mass. This is particularly relevant for young women wishing to preserve childbearing potential ${ }^{7}$ or for elderly women to avoid systematic removal of adnexal masses. Our results confirm that papillary projections, an intermediate $\mathrm{T} 2$ signal intensity within the solid component, ascites and solid portion are predictive of malignancy as previously reported. ${ }^{6}$ In contrast to our findings, Sohaib et $\mathrm{al}^{3}$ suggest that lesion size is also a predictor of malignancy. This discrepancy is likely due to our selective inclusion of complex and indeterminate masses, which did not include small purely cystic benign lesions. In our experience, high DW signal intensity within the solid component is also predictive of malignancy. Nevertheless, high DW signal intensity has a relative low positive likelihood ratio. Using the combination of intermediate T2 and high DW signal intensity within the solid component, positive likelihood ratio for predicting malignancy increased significantly.

The limitations of our study were firstly, patients who did not undergo surgery with pathology within 4 weeks after MR imaging were excluded in this study. This selection aims to improve radiologic-pathologic correlation but may have induced a potential bias. Secondly, we did not perform a quantitative comparison of the degree of signal intensity on DW images, since signal intensity on MRI is not an absolute value and visual assessment of signal intensity is subject to window level and width.

In addition to morphological characteristics, many tissue parameters such as $\mathrm{T} 1, \mathrm{~T} 2$, perfusion, and diffusion contribute to signal intensity, so MRI is able to identify various types of tissue contained in pelvic masses. ${ }^{8}$ The signal intensity characteristics of ovarian masses make possible a systematic approach to diagnosis. ${ }^{9}$ Magnetic Resonance Imaging (MRI) is an essential problem solving tool to determine the site of origin of a pelvic mass and then to characterize an adnexal mass, especially in patients with indeterminate lesions. MRI is also reliable in 
detecting local invasion. The main advantages of MRI are the high contrast resolution with excellent soft tissue contrast and lack of ionizing radiation exposure, which is particularly important in young female patients. ${ }^{1}$

\section{CONCLUSION}

The most significant criteria for predicting malignancy were intermediate T2 and high DW signal (PLR $=4.33)$ followed by papillary projections (PLR $=3.89$ ). The most significant criteria for excluding malignancy were low DW signal $(N L R=2.88)$ followed by combination of low T2 and low DW signal (NLR $=1.85$ ).

Our study demonstrated that diffusion-weighted MR imaging (DWI) combined with classical T2-weighted imaging is an accurate tool to assess the nature of complex adnexal masses depicted by ultrasonography (US). When a solid component is depicted, our study demonstrated that low T2 signal and low DW signal on diffusion-weighted images of this component are the best criteria for excluding malignancy.

\section{REFERENCES}

1. Foti PV, Attinà $G$, Spadola $S$, et al. MR imaging of ovarian masses: classification and differential diagnosis. Insights into Imaging 2016;7(1):21-41.

2. Sohaib SAA, Reznek RH. MR imaging in ovarian cancer. Cancer Imaging 2007;7(Special issue A):S119-S129.

3. Seung Eun Jung, Jae Mun Lee, Sung Eun Rha, Jae Young Byun, Jung Im Jung, Seong Tai HahnCT and MR Imaging of Ovarian Tumors with Emphasis on Differential Diagnosis. RG 2002;22(6):1305-25.

http://pubs.rsna.org/doi/pdf/10.1148/rg.226025033
4. Timmerman D, Valentin L, Bourne TH, Collins WP, Verrelst $H$, Vergote I. Terms, definitions and measurements to describe the sonographic features of adenexal tumors: a consensus opinion from the International Ovarian Tumor Analysis (IOTA) Group. Ultrasound Obstet Gynecol 2000, 16:500 - 505.

5. Buy JN, Ghossain MA, Sciot C, et al. Epithelial tumors of the ovary: CT findings and correlation with US. Radiology. 1991;178:811-18.

6. Out water EK, Siegelman ES, Talerman A, Dunton C. Ovarian fibromas and cystadenofibromas: MRI features of the fibrous component. J Magn Reson Imaging. 1997;7:465-71.

7. Togashi K, Nishimura K, Kimura I, et al. Endometrial cysts: diagnosis with MR imaging. Radiology.1991;180:73-8.

8. Bazot M, Darai E, Nassar-Slaba J, Lafont C, Thomassin-Naggara I. Value of magnetic resonance imaging for the diagnosis of ovarian tumors: a review. J Comput Assist Tomogr 2008, 32:712 - 723.

9. Jeong $Y$ Y, Outwater E K, Kang H K. Imaging Evaluation of Ovarian Masses. R G 2000; 20 (5): 1445 - 70. http://pubs.rsna.org/doi/pdf/10.1148/radiographics.20.5.g00se101445

Source of Support: Nil. Conflict of Interest: None Declared.

Copyright: (c) the author(s) and publisher. IJMRP is an official publication of Ibn Sina Academy of Medieval Medicine \& Sciences, registered in 2001 under Indian Trusts Act, 1882.

This is an open access article distributed under the terms of the Creative Commons Attribution Non-commercial License, which permits unrestricted non-commercial use, distribution, and reproduction in any medium, provided the original work is properly cited.

Cite this article as: Naima Mannan, Mukesh Mittal. Assessment of General MRI, Morphological and Signal Characteristics of Benign and Malignant Ovarian Tumors. Int J Med Res Prof. 2016; 2(3):158-62. 